\title{
Characterization of Miniature Fiber-Optic Fabry-Perot Interferometric Sensors Based on Hollow Silica Tube
}

\author{
Pinggang JIA ${ }^{1,2}$, Guocheng FANG $^{2}$, and Daihua $\mathrm{WANG}^{3 *}$ \\ ${ }^{1}$ Postdoctoral Research Station of Optical Engineering, Chongqing University, Chongqing, 400030, China \\ ${ }^{2}$ Key Laboratory of Instrumentation Science \& Dynamic Measurement of the Ministry of Education of China, North \\ University of China, Taiyuan, 030051, China \\ ${ }^{3}$ Key Laboratory of Optoelectronic Technology and Systems of the Ministry of Education of China, Chongqing \\ University, Chongqing, 400030, China \\ *Corresponding author: Daihua WANGＥ-mail: dhwang@cqu.edu.cn
}

\begin{abstract}
A miniature fiber-optic Fabry-Perot interferometer (MOFPI) fabricated by splicing a hollow silica tube (HST) with inner diameter of $4 \mu \mathrm{m}$ to the end of a single-mode fiber is investigated and experimentally demonstrated. The theoretical relationship between the free spectrum range and the length of HST is verified by fabricating several MOFPIs with different lengths. We characterize the MOFPIs for temperature, liquid refractive index, and strain. Experimental results show that the sensitivities of the temperature, liquid refractive index, and strain are $16.42 \mathrm{pm} /{ }^{\circ} \mathrm{C},-118.56 \mathrm{~dB} / \mathrm{RIU}$, and $1.21 \mathrm{pm} / \mu \varepsilon$, respectively.
\end{abstract}

Keywords: Fiber-optic; Fabry-Perot interferometer; hollow silica tube; characterization

Citation: Pinggang JIA, Guocheng FANG, and Daihua WANG, "Characterization of Miniature Fiber-Optic Fabry-Perot Interferometric Sensors Based on Hollow Silica Tube," Photonic Sensors, 2016, 6(3): 193-198.

\section{Introduction}

Miniature fiber-optic interferometers have attracted broad attention for their advantages of simple structure, compact size, immunity to electromagnetic interference, high sensitivity and accuracy, and so on. The commonly used configurations for fiber-optic interferometers include Mach-Zehnder interferometers [1-2], Michelson interferometers [3-4], and Fabry-Perot interferometers [5-7]. Compared with the fiber-optic Fabry-Perot interferometers, miniature fiber-optic Fabry-Perot interferometers (MOFPIs) have also been widely applied in the measurement of temperature, strain, pressure, acceleration, displacement, and ultrasound.

The processing method of MOFPIs includes chemical etching $[8,9]$, electrical arc discharge $[10$, 11], laser technology [12], and application of special optical fibers, such as the photonic crystal fiber [13], the tapered optical fiber [14], and the hollow core fiber or hollow silica tube (HST) [15]. Each kind of methods has its special features. For example, chemical etching has low cost but complicated processing. Electric arc discharge is difficult to standardize and repeat. The photonic crystal fiber is high-cost. Compared with these methods, the method using HST has low cost, simple structure, and process without large instruments. Traditional MOFPIs based on the multi-beam inference are

Received: 20 April 2016 / Revised: 8 June 2016

(C) The Author(s) 2016. This article is published with open access at Springerlink.com

DOI: $10.1007 / \mathrm{s} 13320-016-0327-7$

Article type: Regular 
fabricated by inserting two well-cut fibers oppositely into an HST and fusing those together [16], or fusing a section of HST between two single mode fibers (SMF) [17].

In recent years, several miniature fiber-optic interferometers based on wavefront splitting are developed. Sun et al. [18] presented an optical fiber strain and temperature sensor based on an in-line Mach-Zehnder interferometer using thin-core fiber. Huang et al. [19] firstly presented an MOFPI based on HST with small inner diameter. They spliced an HST with 5- $\mu \mathrm{m}$ inner diameter to the tip of an SMF to fabricate a novel MOFPI. Lin et al. [20] demonstrated a broadband MOFPI using an SMF end-spliced with a sphered-end hollow core fiber. Frazao et al. [21] presented an MOFPI by splicing an HST with a $20-\mu \mathrm{m}$ inner diameter to the tip of an SMF. Wang et al. [22] also fabricated a similar structure by applying the chemical etching.

In this paper, an MOFPI fabricated by splicing a hollow silica tube with inner diameter of $4 \mu \mathrm{m}$ to the end of an SMF is investigated and experimentally demonstrated. The theoretical relationship between the free spectrum range and the length of HST is verified by fabricating several MOFPIs with different lengths. The MOFPIs for temperature, liquid refractive index, and strain are characterized.

\section{Configuration and operating principle}

The configuration and operating principle of the proposed MOFPI are illustrated in Fig. 1, and the longitudinal and cross-sectional microscopy images of fabricated MOFPI are shown in Fig. 2. The fabrication process of the MOFPIs is as follows. First, a well-cut SMF (Corning SMF-28, 9/125) and an HST are placed in the left and right holder of fusion splicer (FITE, S183 Version2, Japan). Second, set the intensity and time of the discharge of the fusion splicer as 100 unit and $285 \mathrm{~ms}$, and operate arc discharge once. Finally, cut off the HST at an appropriate distance from the fusion joint under the microscope. According to Fig. 2, the inner and outer diameters of HST are approximately $4 \mu \mathrm{m}$ and
$125 \mu \mathrm{m}$. When lights propagate along SMF and get to its end, a part of lights in optical fiber core are reflected. Because the inner diameter of HST is smaller than the core diameter of SMF, the remaining lights propagate into HST and are reflected at the end of HST. Two parts of the lights interfere and form Fabry-Perot interference.

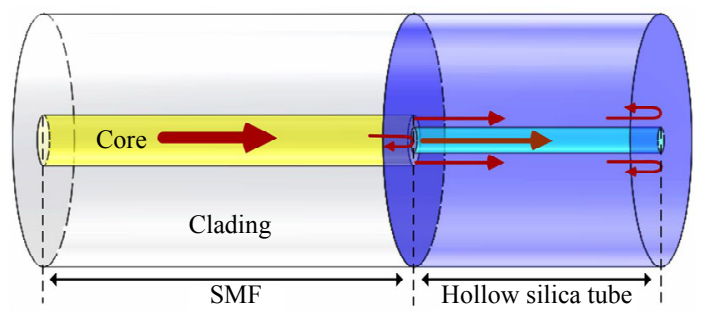

Fig. 1 Configuration of MOFPI.

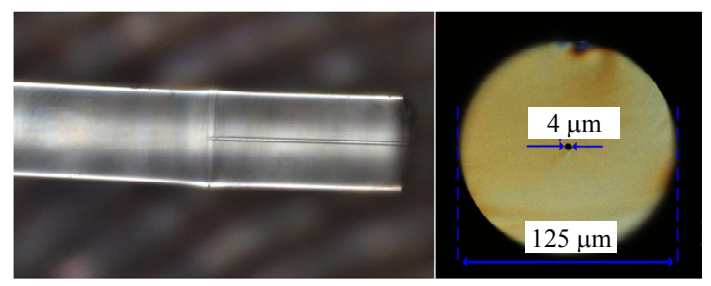

Fig. 2 Longitudinal and cross-sectional microscopy images.

The light can be treated as an electromagnetic wave with time-varying electric fields. The electric field of the reflective lights from the ends of optical fiber and HST can be given by

$$
\begin{aligned}
E_{1} & =r \eta E_{0} \cos \left(\omega t-k x_{1}+\phi_{0}\right) \\
E_{2} & =r(1-\eta) E_{0} \cos \left(\omega t-k x_{2}+\phi_{0}\right)
\end{aligned}
$$

where $E_{0}$ is the electric field of the incident lights, $r$ is the reflective coefficient of the interface of air-silica, $\eta$ is the energy ratio of lights at the facets of fiber-air and fiber-HST, $\omega$ is the angular frequency, $t$ is the time, $x_{1}$ and $x_{2}$ are the positions of the propagation, $k$ is the propagation constant, and $\phi_{0}$ is the phase constant.

The intensity of reflective lights can be given by

$$
\begin{aligned}
I & =I_{1}+I_{2}+2 \sqrt{I_{1} I_{2}} \cos \phi \\
& =r^{2} E_{0}^{2}\left[\eta^{2}+(1-\eta)^{2}+2 \eta(1-\eta) \cdot \cos \phi\right]
\end{aligned}
$$

where $I_{1}$ and $I_{2}$ are intensities of reflective lights from the ends of optical fiber and HST, respectively. $\phi=4 \pi n L / \lambda$, where $n$ is the refractivity of silica, $L$ is the length of HST, and $\lambda$ is the wavelength of 
the light.

The simulation result is shown in Figs.3(a) and (b) according to (3) using the software of MATLAB, when $\eta=0.45, r^{2}=0.037$ and $L=168 \mu \mathrm{m}$ and $527 \mu \mathrm{m}$.

The interference spectrums of the MOFPIs are obtained by the optical spectrum analyzer (Micron Optics Inc, SM125, USA). The interference spectra are illustrated in Fig. 3 when the lengths of the HSTs are $168 \mu \mathrm{m}, 527 \mu \mathrm{m}$, and $2669 \mu \mathrm{m}$. According to Fig. 3, the interference spectra agree well with the simulation result. The insert loss is about $40 \mathrm{~dB}$, which may be caused by the loss of light at the connections.

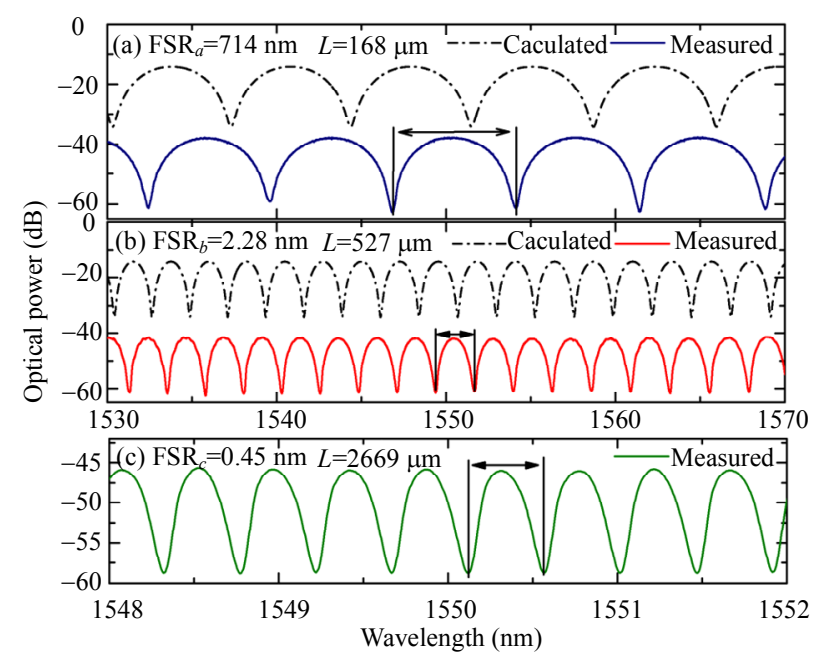

Fig. 3 Interference spectrums of the MOFPIs with HSTs' lengths of (a) $168 \mu \mathrm{m}$, (b) $527 \mu \mathrm{m}$, and (c) $2669 \mu \mathrm{m}$.

The free spectrum range (FSR) can be given by

$$
\mathrm{FSR}=\lambda_{m}^{2} / 2 n L
$$

where $\lambda_{m}$ is the wavelength of light.

The FSRs of the MOFPIs with different lengths of HSTs are fabricated and measured around the wavelength of $1550 \mathrm{~nm}$. The theoretical and measured relationship between the length of HST and FSR are shown in Fig. 4. It can be seen that the measured results agree well with the theoretical results based on (4). The FSRs around the wavelength of $1550 \mathrm{~nm}$ are $7.14 \mu \mathrm{m}$ and $2.28 \mu \mathrm{m}$ when the lengths of the HSTs are $168 \mu \mathrm{m}$ and $527 \mu \mathrm{m}$.

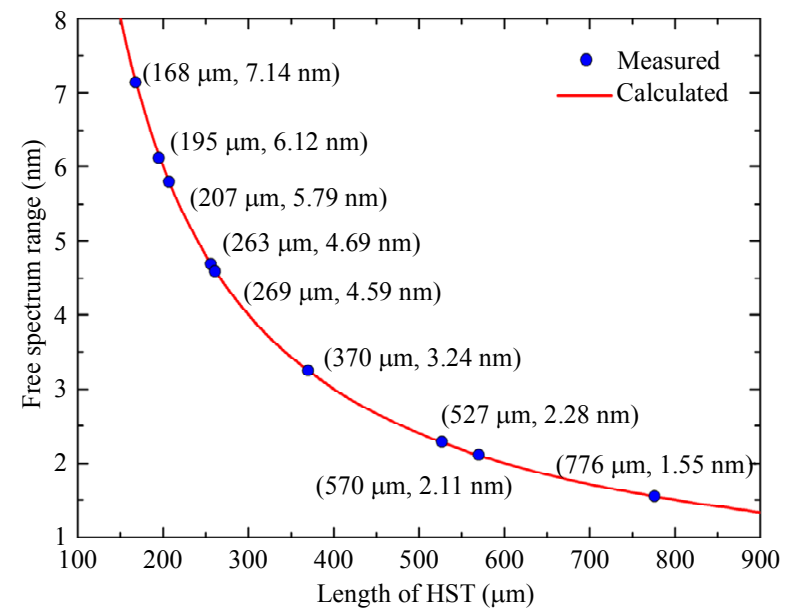

Fig. 4 Relationship between the FSR and the length of HST.

\section{Experiment}

We characterized the MOFPIs for temperature, liquid refractive index (RI), and strain. Regarding the temperature measurements, a sample MOFPI with the length of $263 \mu \mathrm{m}$ was placed inside a controllable muffle furnace (Nabertherm, sn209012, Germany). The sample was hold and protected by stainless steel fixture. We increased the temperature from $25^{\circ} \mathrm{C}$ (room temperature) to $1000{ }^{\circ} \mathrm{C}$ with $50^{\circ} \mathrm{C}$ step. The interference spectrum was recorded when temperature was stabilized of 10 minutes at each step. In the experiment, we used the peak tracing method to demodulate the signal. A wavelength shift of the interference spectrum could be observed with an increasing temperature. The wavelength shift is illustrated in Fig. 5 under the temperature from $25^{\circ} \mathrm{C}$ to $400^{\circ} \mathrm{C}$. The relationship between the wavelength shift of a selected peak and temperature is presented in Fig. 6. The experimental results are well adjusted by the second-order polynomial, given by

$$
y=1552.87+7.72 \times 10^{-3} T+6.61 \times 10^{-6} T^{2} .
$$

The $R^{2}$ (fitting variance) of the fitting curve is $99.88 \%$. However, it is reasonable to divide the temperature range into two different regions, for high $\left(400^{\circ} \mathrm{C}-1000^{\circ} \mathrm{C}\right)$ and low temperatures $\left(25^{\circ} \mathrm{C}-400^{\circ} \mathrm{C}\right.$ ) (insets in Fig. 6), where a much well linear approximation can be done in a high temperature. The sensitivities obtained are 
$16.42 \mathrm{pm} /{ }^{\circ} \mathrm{C}$. The temperature response is caused by the thermal expansion of the material and thermo-optic effect, and the nonlinearity may be caused by the nonlinear change in the thermal expansion coefficient [23].

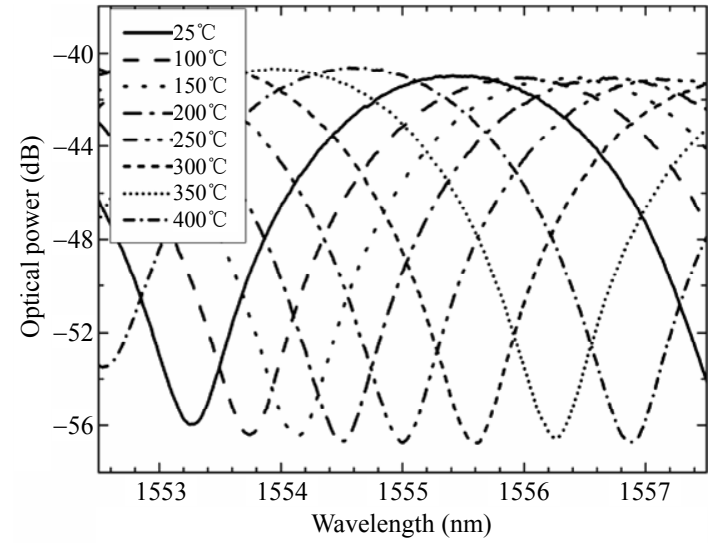

Fig. 5 Wavelength shift of the interference spectrum under the temperature from $25^{\circ} \mathrm{C}$ to $400^{\circ} \mathrm{C}$.

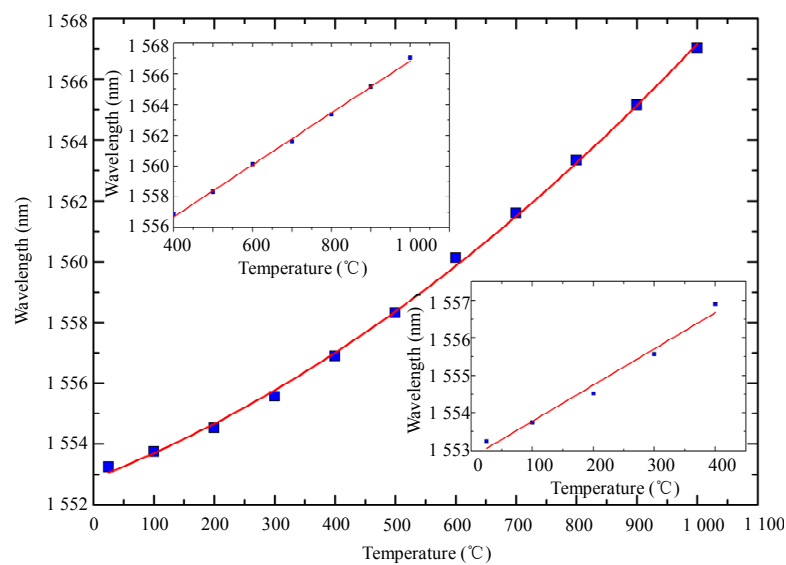

Fig. 6 Temperature response of the sample MOFPI [inset 1 (top left) is the high-temperature response, and inset 2 (bottom right) is the low-temperature response].

In order to test the liquid RI response, a sample MOFPI with the length $L$ of $269 \mu \mathrm{m}$ was vertically inserted in $\mathrm{NaCl}$ solution with different concentrations while the temperature was kept at the room temperature. The RI is related to the concentration of the $\mathrm{NaCl}$ solution and varies from 1.3333 to 1.4069 . The experiment shows that the optical power gradually decreases with an increase in RI, as shown in Fig. 7. The relationship between the optical power of the sample MOFPI and RI is illustrated in Fig. 8. The obtained sensitivity of RI is $-118.56 \mathrm{~dB} / \mathrm{RIU}$.

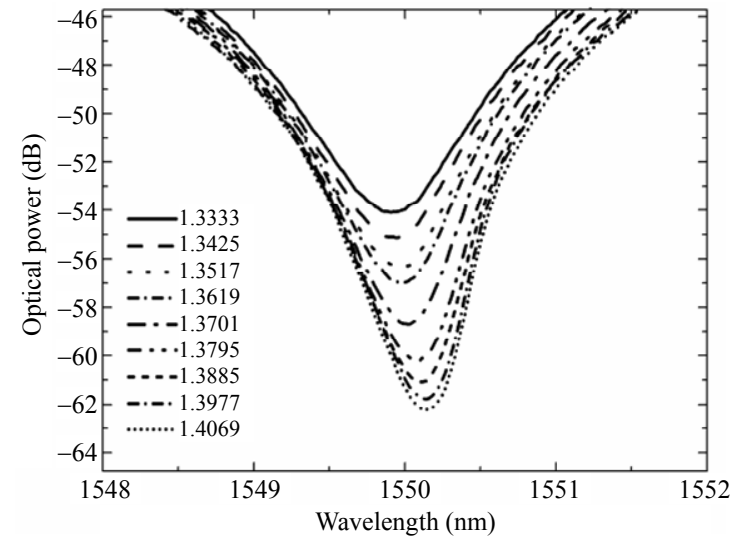

Fig. 7 Interference spectrum when the sample MOFPI was immersed in $\mathrm{NaCl}$ solution with different RIs.

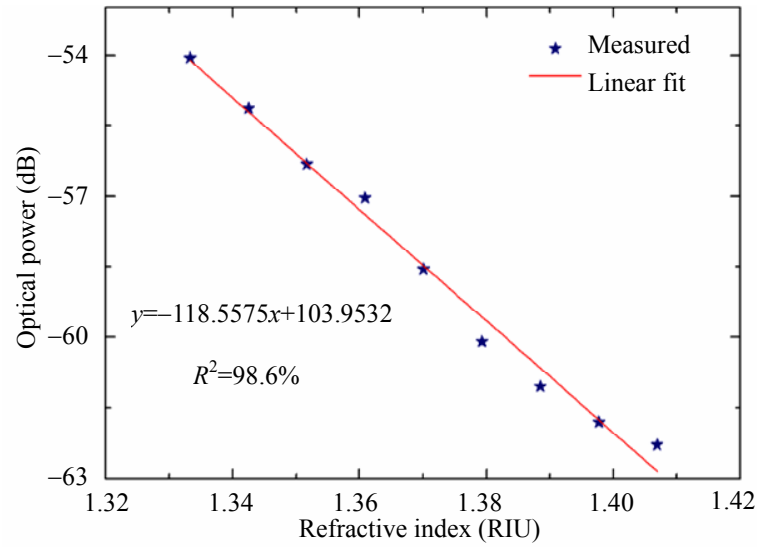

Fig. 8 RI response of the sample MOFPI.

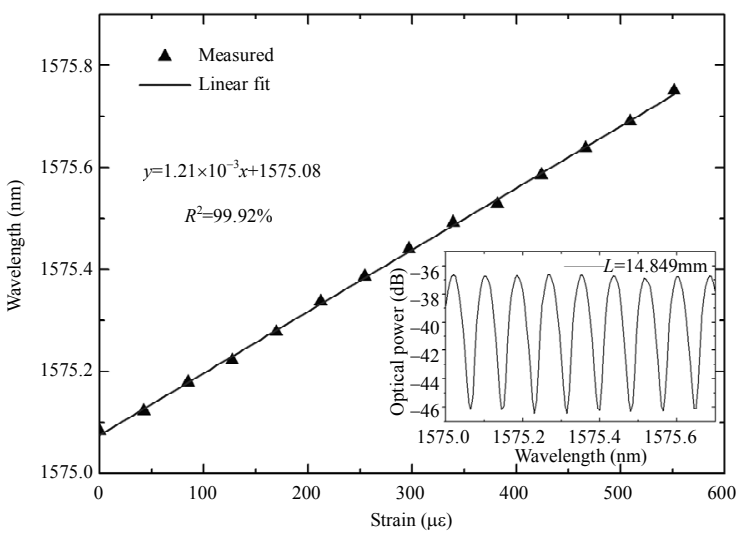

Fig. 9 Strain response of the sample MOFPI [inset (bottom right) is the interference spectrum at around the wavelength of $1575 \mathrm{~nm}]$

In order to test its response to strain, we fabricated an MOFPI with the length of $14.849 \mathrm{~mm}$, whose interference spectrum is shown in Fig. 9 inset. The FSR of the sample is about $83.5 \mathrm{pm}$ at around the wavelength of $1575 \mathrm{~nm}$. We fixed the sample with strain adhesive (KYOWA, \#2129, Japan) on a constant-strength brass beam to test strain. A 
wavelength shift of the interference spectrum was observed when the strain increased, which was caused by the length change of the HST. The result is shown in Fig. 9 and $R^{2}$ of the fitting curve is 99.55\%. The sample shows a sensitivity of $1.21 \mathrm{pm} / \mu \varepsilon$ under the range of $0 \mu \varepsilon-550 \mu \varepsilon$. So the MOFPI can be applied in the measurement of strain.

\section{Conclusions}

In conclusion, an MOFPI fabricated by splicing a hollow silica tube (HST) with an inner diameter of $4 \mu \mathrm{m}$ and outer diameter of $125 \mu \mathrm{m}$ to the end of SMF was investigated and experimentally demonstrated. The theoretical relationship between the free spectrum range and the length of HST was verified by fabricating several MOFPIs with different lengths. We characterized the MOFPIs for temperature, liquid refractive index, and strain. Experimental results showed that the sensitivities of the temperature, liquid refractive index, and strain are $16.42 \mathrm{pm} /{ }^{\circ} \mathrm{C},-118.56 \mathrm{~dB} / \mathrm{RIU}$, and $1.21 \mathrm{pm} / \mu \varepsilon$, respectively. Due to its simple fabrication process and low cost, the MOFPI was suitable for mass production. Experimental results showed that the device had a good response to temperature, liquid refractive index, and strain. The MOFPI had advantages of compact size, simple structure and process, and sensitivity to multi-parameters.

\section{Acknowledgment}

This work is supported by the National Natural Science Foundation of China under Grant 81127901 and 51405454, and Natural Science Foundation of Shanxi Province under Grant 2015021087.

Open Access This article is distributed under the terms of the Creative Commons Attribution 4.0 International License (http://creativecommons.org/licenses/by/4.0/), which permits unrestricted use, distribution, and reproduction in any medium, provided you give appropriate credit to the original author(s) and the source, provide a link to the Creative Commons license, and indicate if changes were made.

\section{References}

[1] W. Talataisong, D. N. Wang, R. Chitaree, C. R. Liao, and C. Wan, "Fiber in-line Mach-Zehnder interferometer based on an inner air-cavity for high-pressure sensing," Optics Letters, 2015, 40(7): 1220-1222.

[2] B. Sun, Y. J. Huang, S. Liu, C. Wang, J. He, C. R. Liao, et al., "Asymmetrical in-fiber Mach-Zehnder interferometer for curvature measurement," Optics Express, 2015, 23(11): 14596-14602.

[3] L. B. Yuan, J. Yang, Z. H. Liu, and J. X. Sun, "In-fiber integrated Michelson interferometer," Optics Letters, 2016, 31(18): 2692-2694.

[4] A. Zhou, G. P. Li, Y. H. Zhang, Y. Z. Wang, C. Y. Guan, J. Yang, et al., "Asymmetrical Twin-core fiber based Michelson interferometer for refractive index sensing," Journal of Lightwave Technology, 2011, 29(19): 2985-2991.

[5] S. Pevec and D. Donlagic, "All-fiber, long-active-length Fabry-Perot strain sensor," Optics Express, 2011, 19(16): 15641-15651.

[6] F. C. Favero, G. Bouwmans, V. Finazzi, J. Villatoro, and V. Pruneri, "Fabry-Perot interferometers built by photonic crystal fiber pressurization during fusion splicing," Optics Letters, 2011, 36(21): 4191-4193.

[7] M. S. Ferreira, J. Bierlich, J. Kobelke, K. Schuster, J. L. Santos, and O. Frazão, "Towards the control of highly sensitive Fabry-Pérot strain sensor based on hollow-core ring photonic crystal fiber," Optics Express, 2012, 20(20): 21946-21952.

[8] X. T. Zou, A. Chao, Y. Tian, N. Wu, H. T. Zhang, T. Y. $\mathrm{Yu}$, et al., "An experimental study on the concrete hydration process using Fabry-Perot fiber optic temperature sensors," Measurement, 2012, 45(5): 1077-1082.

[9] P. A. R. Tafulo, P. A. S. Jorge, J. L. Santos, O. Frazão, "Fabry-Pérot cavities based on chemical etching for high temperature and strain measurement," Optics Communications, 2012, 285(6): 1159-1162.

[10] S. Liu, Y. P. Wang, C. G. Liao, G. J. Wang, Z. Y. Li, Q. Wang, et al., "High-sensitivity strain sensor based on in-fiber improved Fabry-Perot interferometer," Optics Letters, 2014, 39(7): 2121-2124.

[11] D. W. Duan, Y. J. Rao, Y. S. Hou, and T. Zhu, "Microbubble based fiber-optic Fabry-Perot interferometer formed by fusion splicing single-mode fibers for strain measurement," Applied Optics, 2012, 51(8): 1033-1036.

[12] Y. Liu and S. L. Qu, "Optical fiber Fabry-Perot interferometer cavity fabricated by femtosecond laser-induced water breakdown for refractive index sensing," Applied Optics, 2014, 53(3): 469-474.

[13] T. T. Wang and M. Wang, "Fabry-Pérot fiber sensor 
for simultaneous measurement of refractive index and temperature based on an in-fiber ellipsoidal cavity," IEEE Photonics Technology Letters, 2012, 24(19): 1733-1736.

[14] S. C. Gao, W. G. Zhang, Z. Y. Bai, H. Zhang, W. Lin, L. Wang, et al., "Microfiber-enabled in-line Fabry-Perot interferometer for high-sensitive force and refractive index sensing," Journal of Lightwave Technology, 2014, 32(9): 1682-1688.

[15] G. C. Fang, P. G. Jia, T. Liang, Q. L. Tan, Y. P. Hong, W. Y. Liu, et al., "Diaphragm-free fiber-optic Fabry-Perot interferometer based on tapered hollow silica tube," Optics Communications, 2016, 371: 201-205.

[16] K. Mitchell, W. J. Ebel, and S. E. Watkins, "Low-power hardware implementation of artificial neural network strain detection for extrinsic Fabry-Pérot interferometric sensors under sinusoidal excitation," Optical Engineering, 2011, 6(4): 495-501.

[17] D. H. Wang, S. J. Wang, and P. G. Jia, "In-line silica capillary tube all-silica fiber-optic Fabry-Perot interferometric sensor for detecting high intensity focused ultrasound fields," Optics Letters, 2012, 37(11): 2046-2048.
[18] M. Sun, B. Xu, X. Y. Dong, and Y. Li, "Optical fiber strain and temperature sensor based on an in-line Mach-Zehnder interferometer using thin-core fiber," Optics Communications, 2012, 285(18): 3721-3725.

[19] Z. Huang, X. Chen, Y. Zhu, and A. Wang, "Wavefront splitting intrinsic Fabry-Perot fiber optic sensor," Optical Engineering, 2005, 44(7): 1-3,

[20] N. Chen, K. Lu, J. Shy, and C. Lin, "Broadband Micro-Michelson interferometer with multi-optical-path beating using a sphered-end hollow fiber," Optics Letters, 2011, 36(11): 2074-2076.

[21] M. S. Ferreira, L. Coelho, K. Schuster, J. Kobelke, J. L. Santos, and O. Frazao, "Fabry-Perot cavity based on a diaphragm-free hollow-core silica tube," Optics Letters, 2011, 36(20): 4029-4031.

[22] R. H. Wang and X. G. Qiao, "Intrinsic Fabry-Pérot interferometer based on concave well on fiber end," IEEE Photonics Technology Letters, 2014, 26(14): 1430-1433.

[23] J. Mathew, O. Schneller, D. Polyzos, D. Havermann, R. Carter, W. MacPherson, et al., "In-fiber Fabry-Perot cavity sensor for high-temperature applications, "Journal of Lightwave Technology, 2015, 33(12): 2419-2425. 